\title{
UMBILICAL CHARACTERISTIC NUMBER OF LAGRANGIAN MAPPINGS OF 3-DIMENSIONAL PSEUDOOPTICAL MANIFOLDS
}

\author{
MAXIM È. KAZARIAN \\ Moscow Independent University \\ Altayskaya St., 34-145, Moscow 107 589, Russia \\ E-mail: kazarian@ium.ips.ras.ru
}

\begin{abstract}
As shown by V. Vassilyev [V], $D_{4}^{ \pm}$singularities of arbitrary Lagrangian mappings of three-folds form no integral characteristic class. We show, nevertheless, that in the pseudooptical case the number of $D_{4}^{ \pm}$singularities counted with proper signs forms an invariant. We give a topological interpretation of this invariant, and its applications. The results of the paper may be considered as a 3-dimensional generalization of the results due to V. I. Arnold [A].
\end{abstract}

1. Main theorem. A Lagrangian mapping is defined to be a diagram $L \stackrel{i}{\rightarrow} T^{*} M \stackrel{\pi}{\rightarrow} M$. The first map $i$ in this diagram is an embedding (or immersion) of a smooth Lagrangian submanifold $L$ of the total space $T^{*} M$ of the cotangent bundle over a smooth manifold $M$ (with respect to the natural symplectic structure on $T^{*} M$ given by $\omega=\sum d p_{i} \wedge d q_{i}$, where $q_{i}$ are the coordinates of the point $q \in M$ and $p_{i}$ are the corresponding coordinates of the impulse $\left.p \in T_{q}^{*} M\right)$. The second map $\pi: T^{*} M \rightarrow M$ is the natural projection. Throughout the paper we will use the letter $f$ for the composition $\pi \circ i$ and, by a slight abuse of notation, we call sometimes $f: L \rightarrow M$ itself a Lagrangian mapping.

If $L$ is 3-dimensional and generic, all possible pointed singularities are those of types $A_{4}, D_{4}^{+}, D_{4}^{-}$. In general, as shown by $\mathrm{V}$. Vassilyev [V], it is impossible to define an integral invariant of a Lagrangian mapping, which is calculated in terms of $D_{4}^{ \pm}$singularities. Nevertheless it can be done if we restrict ourselves to the class of pseudooptical manifolds.

1.1. Definition. A Lagrangian manifold $L \subset T^{*} M$ is called pseudooptical if there exists a vector field $\xi$ on $L$ whose image on $M$ under $d f$ does not vanish at any point

1991 Mathematics Subject Classification: Primary 57R45; Secondary 53C15.

Research supported by International Science Foundation grant MSD000 and by Russian Foundation for Basic Research (Project Nr. 94-01-01203).

The paper is in final form and no version of it will be published elsewhere. 
of $L$. This vector field $\xi$ will be referred to as the framing or characteristic vector field of the pseudooptical Lagrangian manifold $L$.

For example, an optical (i.e. lying on the level surface of a Hamiltonian which is convex in impulses) Lagrangian manifold is always pseudooptical: for a framing one may take the Hamiltonian vector field. More generally, assume that the generating function $S=\int p d q: L \rightarrow \mathbb{R}$, where $p d q$ is the Liouville 1 -form, has no critical points on $L$; then $L$ is pseudooptical as well: for a framing take the gradient of $S$ (in any metric on $L$ ).

If $L$ is optical there is a natural way to define a sign of every $D_{4}^{ \pm}$singularity (see Definition 2.2 below). Both $D_{4}^{+}$and $D_{4}^{-}$singularities may have both positive and negative signs. We will use the notation $D_{4}^{++}, D_{4}^{+-}$and $D_{4}^{-+}, D_{4}^{--}$, respectively, for these singularities.

We call two pseudooptical immersed Lagrangian submanifolds of $T^{*} M$ isotopic if they may be connected by a continuous family of pseudooptical immersed Lagrangian submanifolds with framing continuously depending on the parameter and such that the caustic (the set of critical values of the Lagrangian mapping) of every manifold of the family is compact.

1.2. THEOREM. The algebraic number of $D_{4}^{ \pm}$singularities of a pseudooptical 3 -fold $L$ counted with their signs

$$
d(L)=\# D_{4}^{++}-\# D_{4}^{+-}+\# D_{4}^{-+}-\# D_{4}^{--}
$$

is an invariant of the isotopy class of immersed pseudooptical Lagrangian submanifolds of $T^{*} M$.

1.3. EXAMPLE. The standard Lagrangian cylinder $([\mathrm{A}])$ is a Lagrangian submanifold $L_{0}$ of the standard symplectic space $\mathbb{R}^{6}=T^{*} \mathbb{R}^{3}$ diffeomorphic to $\mathbb{R} \times S^{2}$ and given by

$$
L_{0}=\left\{(p, q) \in \mathbb{R}^{6}|| p \mid=1, q=s p, s \in \mathbb{R}\right\} .
$$

The standard Lagrangian cylinder is obviously pseudooptical (with framing $\partial / \partial s$ ) and its caustic (just one point) is compact. Let $L \subset T^{*} \mathbb{R}^{3}$ be a generic pseudooptical Lagrangian manifold isotopic to $L_{0}$.

1.4. Proposition. $d(L)=4$. In particular, the caustic of $L$ has at least 4 points of singularity $D_{4}^{ \pm}$.

1.5. Notes. 1. We do not know if there are any topological obstacles to continuous extension of a framing when deforming a given Lagrangian submanifold.

2. Though we give in Section 8 some higher-dimensional analog to the invariant $d(L)$ we failed to find its reasonable applications. Thus, the number 4 in Proposition 1.4 and that in Arnold's symplectic interpretation of the problem about 4 vertices of a convex plain curve $[\mathrm{A}]$ seem to have different nature.

3. Results similar to 1.2 were found by Yu. Chekanov and V. Zakalyukin independently. In fact, the definition of the invariant $d(L)$ (for the optical case) is implicitly contained in $[\mathrm{Ch}]$.

The proof of the main result of the paper, Theorem 1.2, is given in Section 2; the rest of the paper may be considered as comments to this theorem. 
Acknowledgements. The author is grateful to V. I. Arnold for putting the problem. The author thanks also V. M. Zakalyukin, I. R. Porteous and V. A. Vassilyev for stimulating conversations and uses this opportunity to express his gratitude to the Institut für Mathematik Ruhr-Universität-Bochum for its hospitality and support.

2. Proof of Theorem 1.2. A Lagrangian $D_{4}^{ \pm}$singularity is a germ of Lagrangian mapping of 3 -folds given by the germ of the generating family (see, for example, [AG])

$$
F\left(x, y ; q_{1}, q_{2}, q_{3}\right)=x^{2} y \pm y^{3}+q_{1} x+q_{2} y+q_{3} y^{2}
$$

as follows:

$$
L=\left\{(p, q) \in T^{*} M \mid \exists(x, y), \frac{\partial F}{\partial x}=\frac{\partial F}{\partial y}=0, p_{i}=\frac{\partial F}{\partial q_{i}}, i=1,2,3\right\} .
$$

$D_{4}^{ \pm}$singularities called umbilical singularities appear as a type of possible stable singularities of Lagrangian mappings of 3 -folds. Singularities of caustics (critical values of $f)$ at $D_{4}^{+}$and $D_{4}^{-}$points are called also purse and pyramid respectively.

$D_{4}^{ \pm}$singularities may be characterized by the condition that at the corresponding point the kernel of the tangent map $d f: T_{m} L \rightarrow T_{f(m)} M, m \in L$, is 2-dimensional. The one-dimensional image $\operatorname{Im} d f\left(T_{m} L\right) \subset T_{f(m)} M$ is called the tangent line to the caustic singularity $D_{4}^{ \pm}$.

The tangent line to the purse is that to its cuspidal edge and the tangent line to the pyramid is the common one to its three cuspidal edges. The two branches of the cuspidal edge of the purse are essentially distinguishable: one of them consists of points of singularities of type $A_{3}^{+}$and the other of those of type $A_{3}^{-}$. In the same way for the case of pyramid we have three branches of cuspidal edges of type $A_{3}^{+}$on one side of the $D_{4}^{-}$ singularity and three of type $A_{3}^{-}$on the other. Thus, the tangent line to the caustic $D_{4}^{ \pm}$ carries a natural orientation. We choose this orientation in such a way that the orienting vector is directed along the cuspidal edges of type $A_{3}^{-}$. As an orienting vector of the tangent line to the caustic given by the generating family 2.1 may be taken the one given by $\partial / \partial q_{3}$.

Suppose that the Lagrangian manifold $L$ is pseudooptical. Then the image under $d f$ of the characteristic vector at a point of Lagrangian $D_{4}^{ \pm}$singularity always lays on the tangent line to the caustic.

2.2. Definition. The sign of a Lagrangian $D_{4}^{ \pm}$singularity on a pseudooptical Lagrangian 3-fold is positive or negative depending on whether or not the orientation of the tangent line to the caustic given by the characteristic vector coincides with the natural one.

The condition of pseudoopticity implies that this sign is correctly defined. Note that the definition of the sign does not depend on the orientation of $L$, moreover, $L$ may even not be orientable at all.

2.3. Pro of of The or em 1.2. The number $d(L)$ may change only at the moments of perestroikas of the caustic. All possible typical perestroikas of caustics in one-parameter families on a 3 -fold are described in $[\mathrm{Z}],[\mathrm{AG}]$. $D_{4}^{ \pm}$singularities taking part in these perestroikas have close tangent lines, so, it is easy to follow their signs. Thus, during the 
perestroikas $D_{4}^{+}$and $D_{4}^{-}$two singularities $D_{4}^{+}$, respectively, $D_{4}^{-}$of opposite signs appear. In the perestroika $D_{5}$ the $D_{4}^{+}$singularity is being exchanged by the $D_{4}^{-}$singularity of the same sign. This completes the proof. Another proof is given in Section 5 .

2.4. Proof of Proposition 1.4. To calculate the value 4 of the invariant $d(L)$ it is enough to calculate it for any small perturbation of $L_{0}$. For a caustic of this perturbation one may take the focal set of the ellipsoid close to the sphere, which has 4 umbilical points; see also Section 4.

3. A note about Maslov index. Let $i: L \rightarrow T^{*} M$ be a typical Lagrangian immersion of a connected 3 -fold and $\Gamma \subset L$ be the set of critical points of the Lagrangian projection $f: L \rightarrow M$. Then (see, for example, [AG]) $\Gamma$ is a smooth surface in $L$ except for points of singularity $D_{4}^{ \pm}$at which $\Gamma$ has conical singularity (diffeomorphic to the germ at the origin of the surface $a^{2}+b^{2}-c^{2}=0$ in the space of triples $\left.(a, b, c)\right)$. A natural coorientation of $\Gamma$ turns it into a cycle. The intersection number of $\Gamma$ with a curve $\gamma$ with ends outside $\Gamma$ is called the Maslov index of the curve $\gamma$.

Fix a point $m_{0} \in L \backslash \Gamma$. Let the subset $L_{k} \subset L \backslash \Gamma$ consist of points $m \in L \backslash \Gamma$ such that the curve connecting $m_{0}$ with $m$ has Maslov index equal to $k$. In fact, the index $k$ takes values in the group $\mathbb{Z}_{\mu_{0}}=\mathbb{Z} / \mu_{0} \mathbb{Z}$ where $\mu_{0}$ is the minimal positive value of the Maslov index on closed curves. In what follows, we assume that $\mu_{0}>1$ (for example, if $L$ is orientable then $\mu_{0}$ is even).

$L \backslash \Gamma$ is a disjoint union of $L_{k}$ for different $k$. Let $\Gamma_{k} \subset \Gamma$ be a part of the critical set $\Gamma$ formed by $\Gamma_{k}=L_{k-1} \cap L_{k}$. Then $\Gamma=\bigcup \Gamma_{k}$ and points of intersection of different $\Gamma_{k}$ and $\Gamma_{l}$ are those of type $D_{4}^{ \pm}$and this intersection is non-empty only if $|k-l|=1$. We say that an umbilical point has Maslov index $k$ if it is an intersection point of $\Gamma_{k}$ and $\Gamma_{k+1}$.

Consider an isotopy $L(t) \subset T^{*} M$ of immersed pseudooptical Lagrangian 3-folds and suppose that for given $k$ and all $t$ at least one of the two components $\Gamma_{k}$ and $\Gamma_{k+1}$ of the critical set $\Gamma$ of $L=L(t)$ is compact and belongs to some compact domain in $T^{*} M$.

3.1. TheOREM. The algebraic number of $D_{4}^{ \pm}$singularities of Maslov index $k$ counted with their signs

$$
d_{k}(L)=\# D_{4}^{++}(k)-\# D_{4}^{+-}(k)+\# D_{4}^{-+}(k)-\# D_{4}^{--}(k), \quad k \in \mathbb{Z}_{\mu_{0}},
$$

is an isotopy invariant.

This theorem generalizes Theorem 1.2; the invariant $d(L)$ is the sum over $k$ of the invariants $d_{k}(L)$.

The proof is similar to that of Theorem 1.2. One should observe only that the Maslov indexes of any two umbilical points taking part in a typical perestroika of caustics in one-parameter families are always the same.

3.2. Example. ([A]; Geodesic flow on Riemannian 3-folds). Let $M$ be a Riemannian manifold diffeomorphic to $S^{3}$ equipped with a generic metric $g$. The metric $g$ considered as a Hamiltonian function on $T^{*} M$ generates the geodesic flow on $M$. Fix a point $q_{0} \in M$. Then the union of all characteristics passing through the unit sphere in $T_{q_{0}}^{*} M$ forms a pseudooptical (in fact, optical) Lagrangian manifold $L$. In this case the part $f\left(\Gamma_{k}\right)$ of the 
caustic is called the $k$ th focal set of the point $q_{0}$. At any umbilical point of the caustic the $k$ th and $(k+1)$ th focal sets meet for some $k$.

3.3. Proposition. For a Lagrangian manifold $L$ as above we have

$$
d(k)= \begin{cases}4, & k \text { odd }, \\ 0, & k \text { even } .\end{cases}
$$

In particular, for $k$ odd, the $k$ th and $(k+1)$ th focal sets meet at least at 4 umbilical points.

Proof. It is enough to calculate the invariants $d_{k}$ for the case when $M$ is a small perturbation of the unit sphere in the Euclidean space. The focal sets of the point $q_{0}$ in this situation are concentrated in neighborhoods of the two poles of the sphere - the point $q_{0}$ and its antipode. The Lagrangian manifold $L$ is locally equivalent to the small perturbation of the standard Lagrangian cylinder defined in Section 1 and we can get the values of the invariants applying Proposition 1.4. In Section 4 we will see that the same result holds for any compact Riemannian 3-fold.

4. Umbilical points of optical Lagrangian manifolds. A Lagrangian manifold $L \subset T^{*} M$ is called optical if it lies on the level surface of a Hamiltonian whose restriction to every fiber of the cotangent bundle is convex. As noticed in [Ch] the sign of a $D_{4}^{-}$point of an optical Lagrangian manifold is always negative: at such a point $m \in L$ the kernel of $d f: T_{m} L \rightarrow T_{f(m)} M$ separates the two components of the cone $\Gamma$, the characteristic vector $\xi$ belongs to the component of $L \backslash \Gamma$ having maximal Maslov index and the curves of singularities $A_{3}^{-}$lie on the component of $\Gamma$ having minimal Maslov index. Therefore, they belong to the different halfspaces bounded by the kernel.

It was also shown in $[\mathrm{Ch}]$ that the characteristic vector field is transversal both to the surface $\Gamma$ and to the direction field kerdf along $\Gamma$. The projection of this direction field to $\Gamma$ gives a direction field on $\Gamma$ itself. At a $D_{4}^{ \pm}$point this direction field has a singularity on both components of the cone $\Gamma$.

LEMMA 4.1. The index of the obtained direction field on each of the two components of $\Gamma$ at a $D_{4}^{ \pm}$point is equal to $\pm \frac{1}{2}$ depending on the sign of the $D_{4}^{ \pm}$singularity.

The proof of this lemma given in $[\mathrm{Ch}]$ consists in drawing pictures for the corresponding direction fields. This proof is not complete because were two cases considered only while, in fact, there are three possible types of behavior of this direction field see below.

4.2. Example (Focal sets of convex surfaces). Let $V \subset \mathbb{R}^{3}$ be a convex surface in Euclidean 3-space. Let $n(x)$ be the unit normal vector to $V$ at a point $x \in V$. Consider the mapping $f: V \times \mathbb{R} \rightarrow \mathbb{R}^{3},(x, t) \mapsto x+t n(x)$. In fact, this mapping is Lagrangian, and its caustic is called the focal set of $V$. The optical Lagrangian manifold of this mapping is the union of all characteristics $l(x)$ of the Hamiltonian $H=p^{2}$ passing through covectors $n(x), x \in V$. Every characteristic $l\left(x_{0}\right)$ intersects the critical set $\Gamma$ at two (possibly coinciding) points, the corresponding values of $t$ being equal to the values of the two main curvatures of the surface at $x_{0}$.

$D_{4}^{ \pm}$singularities of the focal set correspond to umbilical points of $V$ where both main curvatures coincide. The projection of the direction field ker $d f$ on the two branches of 
$\Gamma$ along the characteristics gives two direction fields on $V$ which coincide with the two fields of directions of the main curvatures on $V$. Singularities of this direction field are studied in $[\mathrm{D}],[\mathrm{P} 1],[\mathrm{P} 2]$. The three possible types of behavior of this direction field are called lemon, (le)monstar and star. $D_{4}^{+-}$singularities and $D_{4}^{--}$are always of star form (with index $-\frac{1}{2}$ ), while $D_{4}^{++}$singularities may be of either lemon or monstar form (with index $\left.\frac{1}{2}\right)$.

Interpretation of the Euler characteristic of a compact manifold as the sum of indexes of a vector (or direction) field on it gives

4.3. Proposition. Suppose that the component $\Gamma_{k}$ of the optical Lagrangian manifold $L$ is compact. Then

$$
d_{k-1}(L)+d_{k}(L)=2 \chi\left(\Gamma_{k}\right) .
$$

Summing these formulae over all $k$ gives the formula due to Chekanov $[\mathrm{Ch}] \chi(\Gamma)=$ $d(L)-\# D_{4}^{ \pm}=-2 \# D_{4}^{ \pm-}, \Gamma$ being compact. If the manifold $L$ is simply connected then every characteristic meets $\Gamma_{k}$ at a unique point. Hence, $\Gamma_{k}$ is homeomorphic to the space of characteristics. Thus, under the hypotheses of Propositions 1.4 and 3.3 we have $\Gamma_{k} \sim S^{2}$, and $d_{k-1}(L)+d_{k}(L)=4$. So, the number 4 in Propositions 1.4 and 3.3 is the doubled Euler characteristic of a 2 -sphere.

4.4. Corollary. The $k$ th and $(k+1)$ th focal sets of any point in any 3-dimensional manifold with respect to any generic Riemannian metric meet at 4 (for odd $k$ ) and 0 (for even $k$ ) umbilical points, counted with their signs.

5. Geometric interpretation of the invariant. Consider a Lagrangian 3-fold $L$ in the standard symplectic space $T^{*} \mathbb{R}^{3}=\mathbb{R}^{6}$ with coordinates $\left(p_{i}, q_{i}\right)$. Denote by $\Lambda_{3}^{+}$ the manifold of all oriented Lagrangian subspaces of $\mathbb{R}^{6}$. The Gauß mapping $G: L \rightarrow \Lambda_{3}^{+}$ assigns to every point $m \in L$ the tangent plane $T_{m} L \subset \mathbb{R}^{6}$. Let $V \subset \mathbb{R}^{6}=T^{*} \mathbb{R}^{3}$ be the "vertical" plane $T_{0}^{*} \mathbb{R}^{3}=\{q=0\}$. Put

$$
D=\left\{\lambda \in \Lambda_{3}^{+} \mid \operatorname{dim} \lambda \cap V \geq 2\right\} .
$$

The Lagrangian projection of $L$ has $D_{4}^{ \pm}$singularity at a point $m \in L$ if and only if $G(m) \in D$. The set $D \subset \Lambda_{3}^{+}$is smooth of codimension 3, except for a subset of codimension 3 in $D$. Hence, $D$ defines a cycle of codimension 3 . Unfortunately, $D$ is not coorientable and the intersection index with $D$ is defined only modulo 2 .

5.1. Definition. The framed Lagrangian Grassmannian $F \Lambda_{3}^{+}$is the set of pairs $(\lambda, \xi)$ where $\lambda \subset \mathbb{R}^{6}$ is an oriented Lagrangian subspace and $\xi \in \lambda$ is a vector transversal to the fixed Lagrangian plane $V \subset \mathbb{R}^{6}$.

Let $\widetilde{D} \subset F \Lambda_{3}^{+}$be formed by the set of pairs $(\lambda, \xi)$ such that $\operatorname{dim} \lambda \cap V \geq 2$. Again, $\widetilde{D}$ is a cycle of codimension 3 in $F \Lambda_{3}^{+}$.

5.2. Proposition. The cycle $\widetilde{D}$ has a natural coorientation.

P r o of. Consider a curve $(\xi(t), \lambda(t))$ on $F \Lambda_{3}^{+}$. Set $K=\lambda(0) \cap V$ and assume that $(\xi(0), \lambda(0))$ belongs to the smooth part of $\widetilde{D}$, i.e. $\operatorname{dim} K=2$. The curve $\lambda(t)$ may be generated by a Hamiltonian flow with a quadratic Hamiltonian function. Calculations 
show that the curve $(\xi(t), \lambda(t))$ touches $\widetilde{D}$ exactly when the restriction of the Hamiltonian to $K$ vanishes. This gives the isomorphism

$$
T_{(\lambda(0), \xi(0))} F \Lambda_{3}^{+} / T_{(\lambda(0), \xi(0))} \widetilde{D} \cong S^{2}\left(K^{*}\right) .
$$

The orientation of $\lambda(0)$ and the vector $\xi(0)$ define an orientation on $K$ and, hence, that on $S^{2}\left(K^{*}\right)$. This gives the desired coorientation of $\widetilde{D}$.

5.3. Note. Curves on $D$ changing coorientation of $D$ are those changing orientation of 2-dimensional $\lambda \cap V, \lambda \in D$. In the case of $\widetilde{D}$ the orientation of $\lambda \cap V$ is defined by the vector $\xi,(\xi, \lambda) \in \widetilde{D}$. This gives another proof of the Proposition.

Let now $L$ be a pseudooptical Lagrangian submanifold of $T^{*} \mathbb{R}^{3}$. Let $\widetilde{G}: L \rightarrow F \Lambda_{3}^{+}$ map a point $m \in L$ to the pair (tangent plane to $L$ at $m$, the characteristic vector at $m$ ). The following proposition is proved by straightforward calculations.

5.4. Proposition. The sign of the Lagrangian singularity $D_{4}^{ \pm}$defined in Section 2 coincides with the sign of the local intersection index of $\widetilde{G}(L)$ with the cycle $\widetilde{D}$.

Note that a change of the orientation of $L$ implies both a change of the orientation of $\widetilde{G}(L)$ and a change of the coorientation of $\widetilde{D}$. Hence, the local intersection index of $\widetilde{G}(L)$ and $\widetilde{D}$ does not depend on the orientation of $L$.

5.5. It is interesting to calculate the cohomology group of $F \Lambda_{3}^{+}$. The construction of symplectic reduction in the skew-orthogonal hyperplane to $\xi$ shows that $F \Lambda_{3}^{+}$is homotopy equivalent to the total space of the bundle with base $S^{2}$ and fiber $\Lambda_{2}^{+} \sim S^{2} \times S^{1}$. It is not difficult to construct a cell division of $F \Lambda_{3}^{+}$with comparatively few cells. Calculations show that $\mathrm{F \Lambda}_{3}^{+}$has the same integral cohomology group as $S^{2} \times S^{2} \times S^{1}$ (at least as $\mathbb{Z}$-module). It is seen also from calculations that the cycle $\widetilde{D}$ is a disjoint union of two homological cycles, $\widetilde{D}=\widetilde{D}^{\prime} \sqcup \widetilde{D}^{\prime \prime}$. It follows that

$$
d(L)=d^{\prime}(L)+d^{\prime \prime}(L)
$$

where $d^{\prime}(L)$ and $d^{\prime \prime}(L)$ are the intersection numbers of $\widetilde{G}(L)$ with $\widetilde{D}^{\prime}$ and $\widetilde{D}^{\prime \prime}$ respectively. Moreover,

5.6. Corollary. If $L$ is orientable and compact then $d^{\prime}(L)=d^{\prime \prime}(L)$ and hence $d(L)$ is always even.

To determine whether a given $(\lambda, \xi) \in \widetilde{D}$ belongs to $\widetilde{D}^{\prime}$ or to $\widetilde{D}^{\prime \prime}$ one should perturb a little $\lambda$ to $\tilde{\lambda}$ with the help of a negative definite Hamiltonian. Then $(\lambda, \xi) \in \widetilde{D}$ belongs to $\widetilde{D}^{\prime}$ or to $\widetilde{D}^{\prime \prime}$ depending on whether or not the orientation of $\tilde{\lambda}$ coincides with that of $\mathbb{R}^{3}$ under the projection of $\tilde{\lambda} \subset T^{*} \mathbb{R}^{3}$ to $\mathbb{R}^{3}$. (Thus, a Lagrangian mapping of an oriented pseudooptical $L \subset T^{*} \mathbb{R}^{3}$ may have $2^{3}=8$ essentially distinguishable kinds of singularity $D_{4}$ !) In fact, $d^{\prime}(L)$ and $d^{\prime \prime}(L)$ are the sums of $d_{k}(L)$ 's defined in Section 3 with odd end even $k$, respectively.

The standard Lagrangian cylinder $L_{0}$ is not compact. Therefore $d^{\prime \prime}\left(L_{0}\right)=0$ but $d\left(L_{0}\right)=d^{\prime}\left(L_{0}\right)=4$.

6. Lagrangian manifolds given by generating families. A Lagrangian immersion $i: L \rightarrow T^{*} M$ is sad to be given by a generating family of functions if there exists a locally 
submersive mapping of manifolds $\rho: N \rightarrow M$ and a smooth function $F: N \rightarrow \mathbb{R}$ such that $L$ is identified with the non-singular submanifold of $N$ given by the equation: the differential of $F$ along the fibers of $\rho$ equals zero, and the immersion $i$ is given by $i(m)=(p, q) \in T^{*} M$ where $p=d F(m), q=\rho(m)$.

Consider on $L$ the vector bundle $T_{L} N$ and its subbundle $X=\operatorname{ker} d \rho$ of vectors tangent to fibers of $\rho$. The second differential of the restriction of $F$ to fibers of $\rho$ at a point $m \in L \subset N$ is a well defined quadratic form $s(m)$ on the vector space $X_{m}$.

Suppose that $L$ and $M$ are 3-dimensional and fibers of $\rho$ are 2-dimensional and oriented. Then Lagrangian $D_{4}^{ \pm}$singularities correspond one-to-one to zeros of the section $s$ of the bundle $S^{2}\left(X^{*}\right)$. This gives

6.1. Proposition. The number of Lagrangian $D_{4}^{ \pm}$singularities counted with proper signs coincides with the intersection number of $s$ with the zero section.

The following construction generalizes the characteristic numbers of $D_{4}^{ \pm}$singularities of both Proposition 6.1 and Section 1.

6.2. Definition. A Lagrangian immersed 3-fold $L \subset T^{*} M$ is called 2-liftable if its tangent bundle $T L$ is represented as a cooriented subbundle of some 5 -dimensional bundle $Y$ over $L$, and there is a submersive mapping of vector bundles $\rho: Y \rightarrow f^{*} T M$ over $L$ such that the restriction of $\rho$ to $T L$ coincides with the tangent map $d f: T L \rightarrow f^{*} T M$.

6.3. EXAmple. A pseudooptical Lagrangian manifold is 2-liftable: for $Y$ one may take the subbundle of $T_{L} T^{*} M$ formed by the hyperplanes skew-orthogonal to the characteristic vectors. A Lagrangian manifold given by a generating family of functions with 2-dimensional fibers is 2-liftable as well: for $Y$ one may take $T_{L} N$, the restriction to $L$ of the tangent bundle of the total space of the generating family.

6.4. ThEOREM. The characteristic number $d(L)$ of Lagrangian $D_{4}^{ \pm}$singularities may be extended to the class of 2-liftable Lagrangian 3-folds.

The proof is essentially the same as the proof of existence of the invariant in all previous cases.

6.5. ExAmple. The standard Lagrangian cylinder $L_{0} \subset T^{*} \mathbb{R}^{3}$ may be given by the generating family

$$
F: S^{2} \times \mathbb{R}^{3} \rightarrow \mathbb{R}, \quad F(v, q)=\langle v, q\rangle, \quad v \in S^{2}, q \in \mathbb{R}^{3},
$$

with fiber $S^{2}$. It follows that any Lagrangian submanifold of $\mathbb{R}^{6}$ close enough to $L_{0}$ may be given by a generating family with fiber $S^{2}$. As an illustration to Theorem 6.4 consider the following result which follows from Propositions 6.1 and 1.4 but can also be proved directly.

6.6. Proposition. Consider a generic family of differential quadratic forms $s_{t}$ on $S^{2}$ ( sections of the vector bundle $S^{2}\left(T^{*} S^{2}\right)$ ) parameterized by $t \in[0,1]$ and assume that the forms $s_{0}$ are negative definite at any point of $S_{2}$ and the forms $s_{1}$ are positive definite at any point of $S_{2}$. Then there are at least 4 points $(v, t)$ in $S^{2} \times[0,1]$ such that the quadratic form $s_{t}$ vanishes at the point $v \in S^{2}$. 
7. Perestroikas of caustics on a plane. Suppose that a Lagrangian manifold $L$ in the standard symplectic space $(p, q) \in \mathbb{R}^{6}$ is transversal to every hyperplane $q_{1}=$ const. Then the gradient of the coordinate function $q_{1}$ restricted to $L$ (in any metric on $L$ ) defines a framing in the sense of Section 1 and we are able to apply our invariant.

7.1. Proposition. Consider a generic family of immersed 2-dimensional Lagrangian submanifolds $L_{t} \subset T^{*} \mathbb{R}^{2}=\mathbb{R}^{4}$ parameterized by $t \in[0,1]$ and assume that the caustic of every $L_{t}$ is compact and belongs to some compact domain of $\mathbb{R}^{2}$. Then the number of perestroikas $D_{4}^{ \pm}$(see $\left.[\mathrm{Z}],[\mathrm{AG}]\right)$ counted with proper signs is an invariant of the family.

8. Some higher-dimensional generalizations. We call a Lagrangian $n$-fold $L \subset$ $T^{*} M k$-liftable if its tangent bundle $T L$ is represented as a cooriented subbundle of codimension $k$ in some vector bundle $Y$ over $L$ and there exists a submersive mapping of vector bundles $\rho: Y \rightarrow f^{*} T M$ such that the restriction of $\rho$ to $T L$ coincides with $d f$ and the subbundle $\operatorname{ker} \rho$ of $Y$ is orientable.

Similar to 6.3 if there exists an $(n-k)$-dimensional orientable subbundle of $T L$ such that the restriction of $d f$ to it is immersive then $L$ is $k$-liftable. Another example of $k$-liftable manifold is that given by a generating family of functions with $k$-dimensional fibers.

For a generic Lagrangian manifold $L \subset T^{*} M$ the set $m_{k}$ of points $x \in L$ at which the tangent mapping $d f: T_{x} L \rightarrow T_{f(x)} M$ has at least $k$-dimensional kernel forms a cycle of codimension $k(k+1) / 2$ (see $[\mathrm{F}])$.

8.1. Theorem. For $k$ odd the cycle $m_{k}$ has a natural coorientation. For $k$ even this cycle is coorientable on k-liftable manifolds.

Note that the coorientation of this cycle does not depend on orientations chosen on $L$ and $M$. Following $[\mathrm{F}]$ we call the cohomology class in $H^{k(k+1) / 2}(L, \mathbb{Z})$ given by the intersection number with $m_{k}$ the Maslov-Arnold characteristic class.

Proof. For $x \in m_{k} \subset L$ set $K=\operatorname{ker} d f: T_{x} L \rightarrow T_{f(x)} M$ and suppose that $x$ is a smooth point of $m_{k}$, that is, $\operatorname{dim} K=k$. Then, similar to the proof of Proposition 5.2 the coorientation of $m_{k}$ is defined by the orientation of the space $S^{2}\left(K^{*}\right)$ of quadratic forms on $K$. For $k$ odd this space has a natural orientation and for $k$ even its orientation is defined by the orientation of the plane $K$ which for $k$-liftable manifolds coincides with the oriented $\operatorname{ker} \rho$.

\section{References}

[A] V. I. Arnold, Sur les propriétés topologiques des projections lagrangiennes en géométrie symplectique des caustiques, Cahiers Math. Décision, CEREMADE, 9320, 14/6/93, 9 pp.

[AG] V. I. Arnold and A. V. Givental, Symplectic Geometry, in: Encyclopedia of Math. Sci. Dynamical Systems 4, Springer, 1990, 4-136.

[Ch] Yu. V. Chekanov, Caustics in geometrical optics, Funct. Anal. Appl. 20 (1986), 223-226. 
[D] G. Darboux, Leçons sur la théorie générale des surfaces, Vol. 4, Gauthier-Villars, Paris, 1896.

[F] D. B. Fuchs, Maslov-Arnol'd characteristic classes, Soviet Math. Dokl. 9 (1968), 96-99.

[P1] I. R. Porteous, The normal singularities of surfaces in $\mathbb{R}^{3}$, in: Proc. Sympos. Pure Math. 40, Part 2, Amer. Math. Soc., 1983, 379-393.

[P2] I. R. Porteous, Geometric Differentiation, Cambridge University Press, 1994.

[V] V. A. Vassilyev, Lagrange and Legendre Characteristic Classes, Gordon and Breach, 1988.

[Z] V. M. Zakalyukin, Rearrangements of fronts and caustics depending on a parameter, and versality of maps, in: Current Problems of Math. 22, VINITI, 1983, 56-93. 